\title{
Effect of Electric Field Strength and Water Content on the Electrorheology of Silica Dispersions
}

\author{
Habauka M. Kwaambwa \\ Faculty of Health and Applied Sciences, Namibia University of Science and Technology, Windhoek, Namibia \\ Email: hkwaambwa@nust.na
}

How to cite this paper: Kwaambwa, H.M. (2017) Effect of Electric Field Strength and Water Content on the Electrorheology of Silica Dispersions. Materials Sciences and Applications, 8, 281-290.

https://doi.org/10.4236/msa.2017.84020

Received: January 2, 2017

Accepted: April 10, 2017

Published: April 13, 2017

Copyright $\odot 2017$ by author and Scientific Research Publishing Inc. This work is licensed under the Creative Commons Attribution International License (CC BY 4.0).

http://creativecommons.org/licenses/by/4.0/

\section{(c) (i) Open Access}

\begin{abstract}
The effect of water content on the electrorheological effect (ERE) behaviour of silica dispersions has been investigated. The types of silica powders were fumed silica, precipitated and acid washed silica and colloidal silica. Silica dispersions with a water content varying in the range $10 \%-30 \%$, and with conducting ions deliberately added, were redispersed in chlorinated hydrocarbon oil and then the ER behaviour studied. Samples were tested on a static yield rig (SYR), an instrument which can measure both the yield stress and the conductivity. The effects of electric field strength and water content on the ERE were studied. The yield stress initially increased with increase in electric field strength and then decreased at high electric fields suggesting a breakdown in structure of the electrorheological fluid samples tested. It was also found that the ERE increased and electric saturation or electric breakdown shifted to lower electric fields as the water content increased. Higher electric fields caused a drop in the ERE.
\end{abstract}

\section{Keywords}

Colloidal Silica, Electrorheological Effect, Electrorheological Fluid, Fumed

Silica, Precipitated and Acid Washed Silica, Static Yield Rig, Yield Stress

\section{Introduction}

Electrorheological (ER) fluid is one kind of "smart" colloid material capable of varying viscosity or solidification in response to an applied electric field. It is basically a suspension with polarisable solid particles dispersed a non-conducting fluid or insulating oil and behaves like a Bingham fluid under an applied electric field. The suspending liquid which should have a high resistivity is normally low viscosity hydrocarbon or silicone oil. The particles dispersed in this 
liquid are commonly metal oxides, alumino silicates, silica, organics or polymers [1]. The change in structure and mechanical properties in the presence of an applied electric field is called the electrorheological effect (ERE) or "Winslow Effect" named after American scholar Willis M. Winslow [2]. When an external field is applied, the dispersed particles are polarized and form chain-like structure. The rheological properties such as apparent viscosity, shear stress, and yield stress are rapidly increased. And when the field is removed, the polarization of the particles disappears and the viscosity quickly returns to its original value. Because of the special and desirable characteristic of the ER fluids, the materials have huge potential for many applications such as dampers, clutches, brakes, robotics and actuators [3] [4]. Because of the diverse applications potential in mechanical elements capable of responding to environmental variationshence the denotation "smart", ER fluids are still attracting great interest in research because their applications are still being hampered by the weakness of the ER effect [5].

The properties of ER fluids depend on particle size, density, carrier fluid properties and temperature [1] [6]. The effect on the ERE of water content in the silica dispersions was studied. It has been widely known in the field of ER fluid research that the presence of any water in the sample can enhance the ER effect, sometimes dramatically [1]. Three types of silica powders used are colloidal silica, precipitated and acid washed silica and fumed silica [7].

The term colloidal silica is referred to stable dispersions or sols of discrete particles of amorphous silica. By arbitrary definition, the term excludes solution of polysilicic acid in which the polymer molecules or particles are so small that they are not stable. Silica gel may be described as a coherent, rigid three-dimensional network of contiguous particles of colloidal silica. Silica powder may consist of small granules of silica gel or of coherent aggregates of submicron particles that are linked together in extremely weak networks. Theoretically, a silica powder might consist of separate, discrete silica particles, but when the particles diameter is less than $100 \mathrm{~nm}$, the particles spontaneously adhere together in loose aggregates. It is only when the discrete particles are much larger, that is, 5 50 microns, that the cohesive forces become so low that the particles do not attract each other and are very "dusty", that is, small enough to form a "smoke" when shaken in air.

Precipitated silica is powder formed when the silica particles are coagulated as loose aggregates in the aqueous medium, recovered, washed and dried. Coagulation may be affected by high salt concentration or other coagulants such as ammonia, water miscible solvents, or certain types of organic materials. When the particles are larger than $5-10 \mathrm{~nm}$, they may be only weakly joined together and if in an open-packed condition subsequently, they can easily be broken apart and dispersed in oils or rubber.

Fumed silica or pyrogenic silica or aerosil is powder made by condensing silica from the vapour phase at elevated temperature. The silica vapour is produced by a) direct volatilisation of silicon dioxide $\left(\mathrm{SiO}_{2}\right)$, b) reduction of $\mathrm{SiO}_{2}$ to vola- 
tile $\mathrm{SiO}$ which is re-oxidised, c) oxidation of volatile silicon compounds such as chloride or esters, or d) vapour phase hydrolysis of $\mathrm{SiF}_{4}$.

\section{Experiment}

\subsection{Preparation of Silica Electrorheological Fluids}

Three types of silica powder were dispersed in high-viscosity chlorinated aliphatic hydrocarbon oil commercially called Cereclor 50LV (FCI plc). The silica powders used were supplied by BDH Chemicals and are colloidal silica, precipitated and acid washed silica, and fumed silica.

Approximately, $10 \mathrm{~g}$ of powder was weighed accurately in a beaker on an analytical balance and made into a paste by adding $1.0 \times 10^{-3} \mathrm{M}$ lithium chloride ( $\mathrm{LiCl})$ as conducting ions [8] and then double-distilled water so that the resulting concentration of $\mathrm{LiCl}$ was about $1.0 \times 10^{-4} \mathrm{M}$ in the aqueous phase. For instance, if $4 \mathrm{~mL}$ of $1.0 \times 10^{-3} \mathrm{M} \mathrm{LiCl}$ was added to the weighed powder $(10 \mathrm{~g}), 36$ $\mathrm{mL}$ of double-distilled water was required. Silica powder dispersed in Cereclor $50 \mathrm{LV}$ in the volume fraction range $10 \%-30 \%$ without added $\mathrm{LiCl}$ with applied fields up to $2.6 \mathrm{kV} \cdot \mathrm{mm}^{-1}$ were examined and no ERE was observed, i.e. preliminary experiments of silica samples prepared as above but without added $\mathrm{LiCl}$ did not exhibit any ERE. Conducting ions were thus deliberately added. The water content (expressed as $\% \mathrm{H}_{2} \mathrm{O}$ ) was varied in the range $10 \%-30 \%$ to study on the ERE. This was done by drying the silica powder paste the oven at $71^{\circ} \mathrm{C}$ until the amount of water remaining in the paste was in the range $10 \%-30 \%$. The amount of water in the paste was accurately derived from separate percentage solids determination, i.e. a small amount of the paste was taken and dried in the oven $105^{\circ} \mathrm{C}$ to constant weight.

The dried paste was then re-dispersed in Cereclor $50 \mathrm{LV}$ using ultrasonification so that a high concentration of silica was obtained. $20 \% \mathrm{w} / \mathrm{v}$ silica dispersions were prepared. About $1 \% \mathrm{w} / \mathrm{w}$ of surface active agent, commercially called Synperonic NP6 (Dow Chemicals) which is anonyl phenol hexaethoxylate, with respect to Cereclor 50LV was added as the dispersing agent as the silica powders would not disperse without it [9]. Samples, especially those for precipitated and acid washed silica, had to be left in the ultrasonic bath as long as 48 hours. The samples were then tested on the shear yield test rig called Static Yield Rig (Laser Engineering (Development) Ltd.).

\subsection{Static Yield Rig (SYR) and Measurement of Samples}

Figure 1 shows the top and side view of a Static Yield Rig (SYR). The SYR is a small laboratory or workshop rig for measuring both the electrorheology and the conductivity of an ER fluid. In this study, the rig was used to measure the electrorheology only to study the effect of electric field strength and water content. The unit is sufficiently accurate to be able to provide details of the zero shear yield behaviour of the fluids. The zero shear yield stress is defined as the stress at which an ER fluid will fail with an electric field applied under conditions of zero 
shear rate.

The rig consists of a fixed high voltage electrode supported in a gantry above a movable lower electrode. The upper electrode is isolated from the gantry by means of an insulated glass filled PTFE bush. Shims under the electrode allow some flexibility in changing the electrode gap. The lower electrode is bonded to a thermoelectric cooler which is in turn bonded to a precision ball slide. Changing the polarity of the thermoelectric cooler converts it into a heater thus permitting temperature control of the rig. The lower electrode is shaped like a trough and the fluid to be tested is poured into the trough. Voltage is applied across the fluid between the two electrodes. Turning the micrometer screw loads the cantilever arm which bears onto the movable lower electrode. The fluid resists and the load is increased until the fluid yields, where upon the slide moves and the reading at maximum stress noted. The unit is reset and the voltage increased. The sequence is repeated.

The cantilever arm has a four arm strain gauge bridge. A strain gauge conditioning amplifier provides the necessary bridge supply as well as the compensation and conditioning for the bridge. The bridge supply is adjustable as the zero offset and the gain. This last feature enables different arms to be used and effects such as creep to be studied.
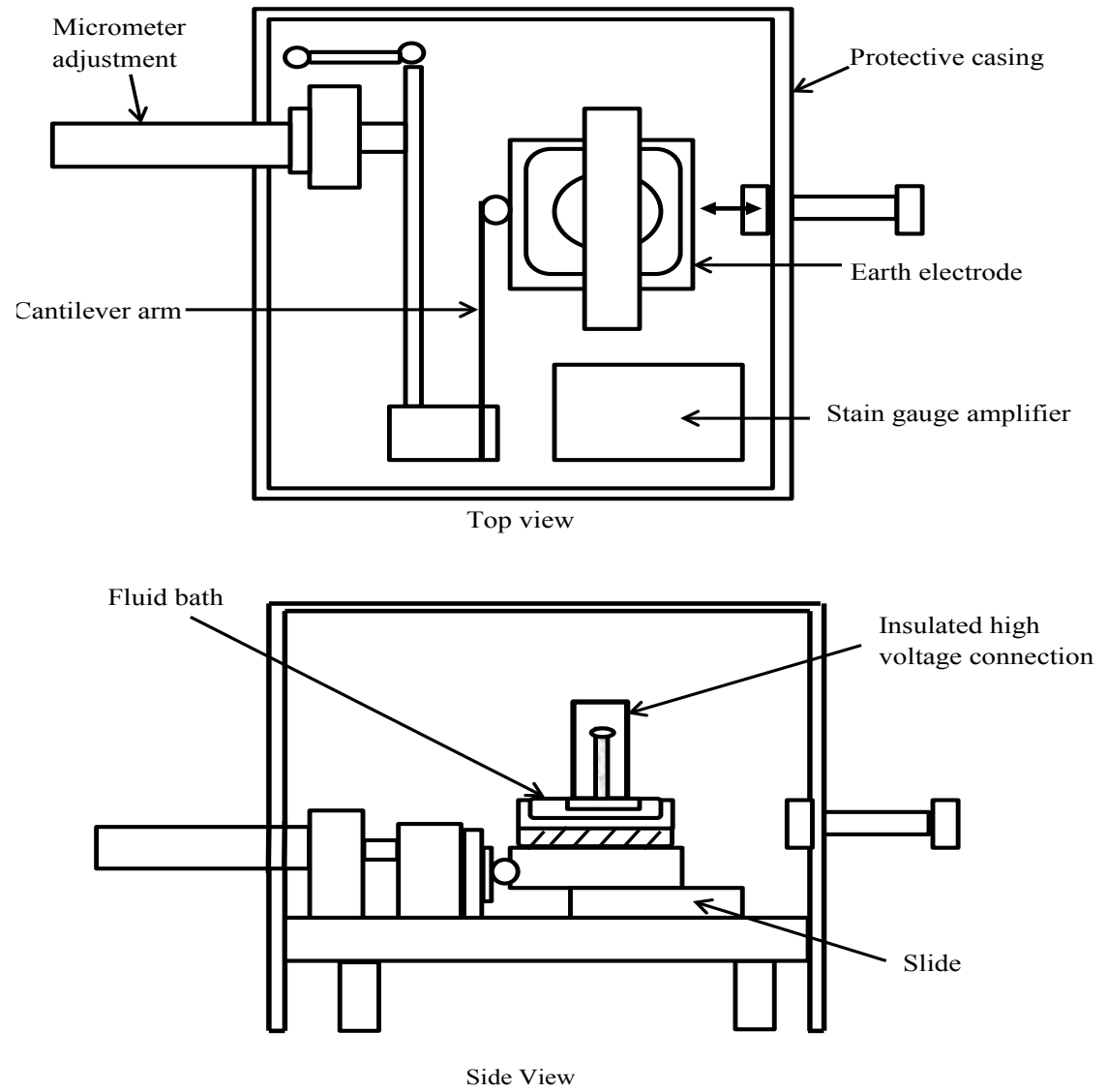

Figure 1. Schematic diagram of the Static Yield Rig. 
The control box (not shown here) is a self-contained measuring unit and houses all the necessary equipment to operate the rig. A temperature controller is fitted as well as a high voltage power supply capable of delivering $2.5 \mathrm{kV}$ at 5.0 $\mathrm{mA}$. This unit is totally short circuit proof and folds back if excessive current is drown. The unit is provided with analogue outputs to monitor both the current and the voltage. These are both $10 \mathrm{~V}$ for full scale deflection. The bridge output is scaled so that that $7.5 \mathrm{~V}$ is equivalent to $20 \mathrm{kPa}$. There is a switch selectable dual display meter, both analogue and digital, provided scaled in volts $0-2.5 \mathrm{kV}$, current 0 - $4 \mathrm{~mA}$ and yield stress 0 - $20 \mathrm{kPa}$. Included are on-off switching, fuseing, heater boost button, display hold button and a high voltage logic interrupt that switches off the voltage. Voltage control is via a 10 turns potentiometer. Four BNC plugs are provided enabling the measured signals (i.e. voltage, current, stress and temperature) to be fed to an external measuring or recording device.

About $4 \mathrm{~mL}$ of the sample fluid was filled into the cell which had been cleaned with acetone. The electric field and water content were studied concurrently for each sample. This was done by increasing the voltage and the yield stress (heights of peaks) recorded. Sometimes it was necessary to change sample before going through the range as soon as the recorder started going out of scale. This could have been due to exposing the sample to electric field for a long time.

\section{Results}

The results for the silica samples electrorheology are shown in Figures 2-4. In general, the yield stress increases with increase in applied electric field up to a certain maximum after which "fluctuations" in the yield stress were observed. The yield stress maximum for a particular type of silica sample increases with increase in water content. Also, the maximum yield stress moves to lower elec-

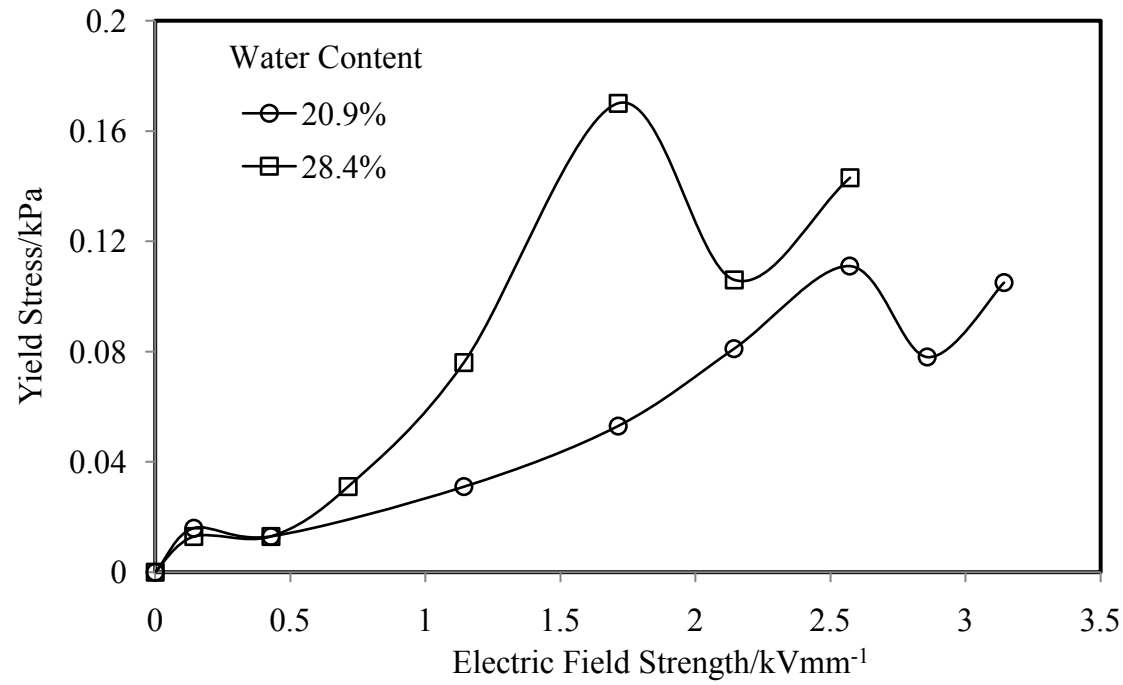

Figure 2. Response of colloidal silica to electric field (Temperature alarm setting: T1 = $35.6^{\circ} \mathrm{C} ; \mathrm{T} 2=36.9^{\circ} \mathrm{C}$ ). 


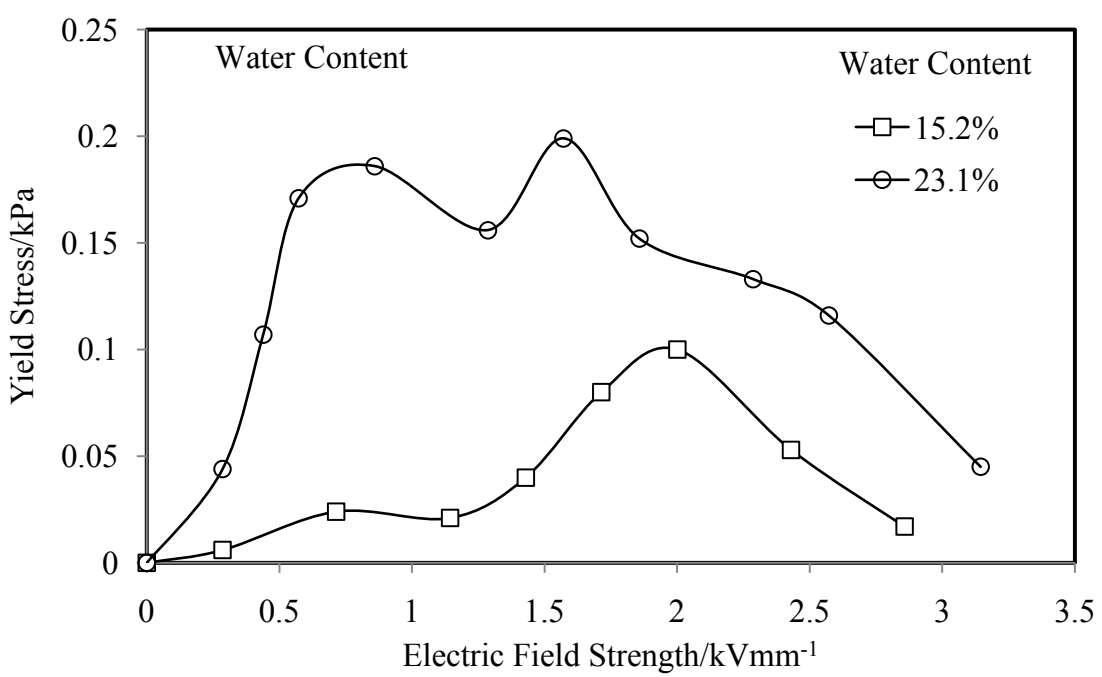

Figure 3. Response of fumed silica to electric field (Temperature alarm setting: $\mathrm{T} 1=$ $35.6^{\circ} \mathrm{C}$; $\left.\mathrm{T} 2=36.9^{\circ} \mathrm{C}\right)$.

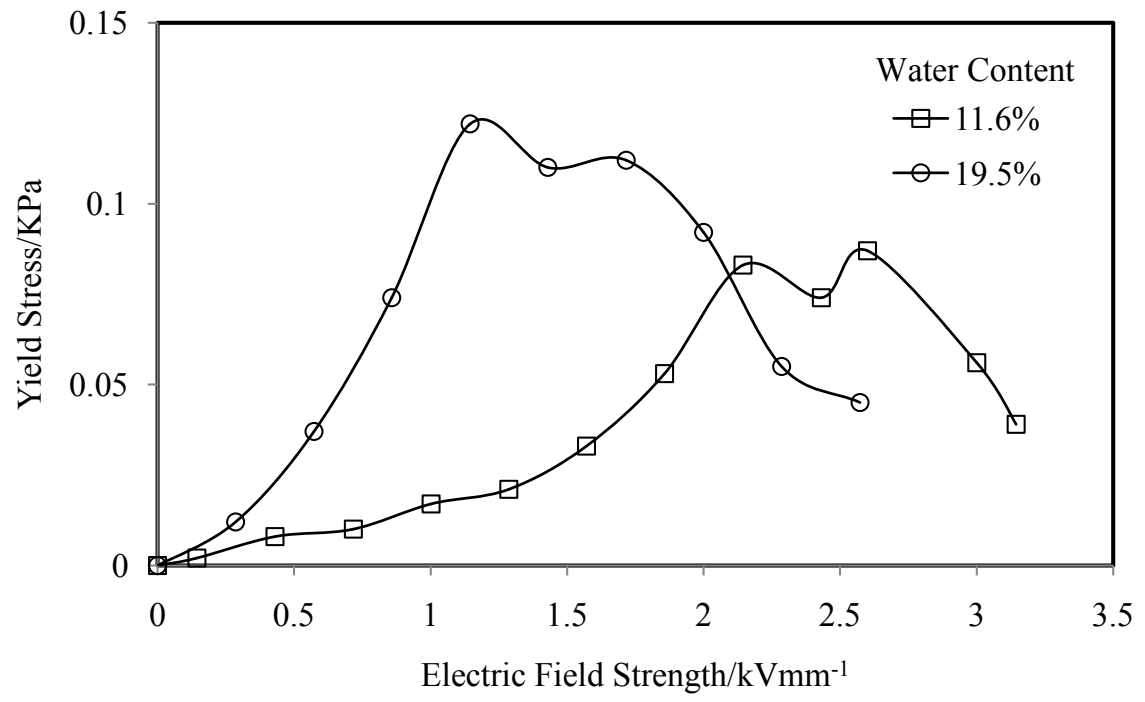

Figure 4. Response of precipitated and acid washed silica to electric field (Temperature alarm setting: $\mathrm{T} 1=35.6^{\circ} \mathrm{C}$; $\mathrm{T} 2=36.9^{\circ} \mathrm{C}$ ).

tric fields as the water content increases, a similar effect was observed by Deinega and Vinogradov [10] for the studies of the influence of hydration of disperse phase in hydrocarbon phase on ERE. In their plot of stress versus water content, they observed that as moisture content increased, the ERE increased, reached a maximum and then fell sharply; the maximum grew and moved towards smaller humidity values. The results here also confirm that the more the electric field was applied, the yield stress dropped. The positions of the maxima and shifts due to water content are given in Table 1. Although estimates were made, it is, however, incorrect to determine and compare the maxima and shifts using electric field values because the curves are non-symmetrical, broad and have fluctuations. It was not possible to determine how much the yield stress increased with 
Table 1. Characteristics of the yield stress versus electric field strength data for silica dispersions extracted from Figures 1-3.

\begin{tabular}{|c|c|c|c|c|c|c|}
\hline \multirow{2}{*}{ Sample } & \multicolumn{2}{|c|}{ Colloidal Silica } & \multicolumn{2}{|c|}{ Fumed Silica } & \multicolumn{2}{|c|}{$\begin{array}{l}\text { Precipitated and } \\
\text { Acid Washed Silica }\end{array}$} \\
\hline & $\begin{array}{c}20.9 \% \\
\mathrm{H}_{2} \mathrm{O}\end{array}$ & $\begin{array}{c}28.4 \% \\
\mathrm{H}_{2} \mathrm{O}\end{array}$ & $\begin{array}{c}15.2 \% \\
\mathrm{H}_{2} \mathrm{O}\end{array}$ & $\begin{array}{c}23.1 \% \\
\mathrm{H}_{2} \mathrm{O}\end{array}$ & $\begin{array}{c}11.6 \% \\
\mathrm{H}_{2} \mathrm{O}\end{array}$ & $\begin{array}{c}19.5 \% \\
\mathrm{H}_{2} \mathrm{O}\end{array}$ \\
\hline Stress at maximum $(\mathrm{kPa})$ & 0.000 & 0.179 & 0.100 & 0.186 & 0.089 & 0.122 \\
\hline $\begin{array}{c}\text { Electric filed at } \\
\text { maximum }(\mathrm{kV} / \mathrm{mm})\end{array}$ & 2.57 & 1.71 & 2.00 & 0.86 & 2.30 & 1.20 \\
\hline $\begin{array}{l}\text { Increase in yield stress } \\
\qquad(\mathrm{kPa})\end{array}$ & \multicolumn{2}{|c|}{0.059} & \multicolumn{2}{|c|}{0.086} & \multicolumn{2}{|c|}{0.033} \\
\hline $\begin{array}{l}\text { Shift to lower electric due } \\
\text { to water content } \\
(\mathrm{kV} / \mathrm{mm})\end{array}$ & \multicolumn{2}{|c|}{0.86} & \multicolumn{2}{|c|}{1.14} & \multicolumn{2}{|c|}{1.10} \\
\hline $\begin{array}{c}\text { Difference in water } \\
\text { content }\left(\% \mathrm{H}_{2} \mathrm{O}\right)\end{array}$ & \multicolumn{2}{|c|}{7.5} & \multicolumn{2}{|c|}{7.9} & \multicolumn{2}{|c|}{8.4} \\
\hline
\end{tabular}

water content as only two samples were tested for a particular silica powder. So, it follows that it is difficult to conclude which silica powder had its yield stress increased more as a result of the increases in water content. What is conclusive, however, is that increasing water content increases the yield stress or ERE.

\section{Discussion}

The application of an electric field to concentrated silica dispersions induces the alignment of dipoles within the particles forming the disperse phase so that the particles possess an induced dipole. This has a number of consequences. In general, the yield stress (ERE) is only weak up to a certain threshold intensity of the electric field. Above this threshold value, the yield stress or ERE raises sharply depending on the water content for a particular type of silica powder. Then there is a tendency to saturation characterized by a number of "fluctuations" in the yield stress in some cases. Finally, the yield stress drops. It is worth noting that not only does the maximum increase with increase in the water content but also moves towards lower electric fields.

\subsection{Effect of Electric Field on ERE}

In hydrocarbon dispersions, the hydrocarbon medium creates favourable conditions for the occurrence of strong electric fields as a result of its low conductance. The thickness of the electric double layer is even larger than in aqueous medium, which is responsible for high deformability [8] [11] [12]. There are several mechanisms for increasing the "electroviscosity" of disperse systems when an external electric field is applied. The "electroviscosity" increase may be related to the orientation of particles, deformation of the electric double layer and the interelectrode oscillation of particles or structural formation. The analysis of individual mechanisms of the ERE shows that the main reason for the considerable increase in the "electroviscosity" of the concentrated disperse systems 
in an electric field is the structure formation [13]. The interaction of the particles is based on dielectric polarisation, i.e. induction of the dipole moments in the disperse particles.

No ERE was observed for preliminary transient measurements on organophilic silica dispersed in hydrocarbon oil Cereclor $50 \mathrm{LV}$ in the volume fraction range $10 \%-30 \%$ with applied field up to $2.6 \mathrm{kV} \cdot \mathrm{mm}^{-1}$. There are several reasons which could be advanced but the probable one is that there was no small and finite conductivity in the particle dispersions studied. Several investigators who have studied ERE phenomena report that a small but finite conductivity is required for the ERE to occur in addition to high water content. Salts like sodium and potassium thiocyanate, potassium perchlorate, lithiumtrifflate and tetrabutylmmoium iodide have been used in polymer based systems and found to increase the yield stress and shear thinning of ER suspensions up to optimum salt concentration such that the magnitude of the ERE correlated with the bulk ionic conductivity of the studied materials [14]. For silica-based ER fluids, the particles prepared by sol-method had to be modified by introducing functional functions such as urea and N, N-dimethylformamide [15] [16] or introducing ions such as $\mathrm{Co}^{2+}$ or $\mathrm{Zr}^{4+}$ [17] for the ERE to be observed. Hence, in this study, $\mathrm{LiCl}$ ions were added as conducting ions [8].

The ERE is attributed to the migration of lithium ions to one side within the spherical particles whereas the chloride ions become part of the negative silica particles. Such species give the particles a very high polarisability and this enables large dipole-dipole interactions to form bridges in a reaction perpendicular to the charged particles providing the field, i.e. along the field lines. The dipole-dipole interactions increase as the electric field increases and so the ERE increases too prior to electrical breakdown.

\subsection{Effect of Water Content on ERE}

The water content has been observed to increase the ERE [1]. The changes of the ERE with increasing water content could be associated with the specific features of polarisation of hydrocarbon disperse systems with a hydrated disperse phase in strong electric fields. The addition of water to silica particles with silanol groups proceeds due to formation of hydrogen bonds. However, no less important for the formation of structure bound water is the formation of hydrogen bonds between the added molecules of water. Such interaction results in the formation of chain structures of water on the surface and in pores. The counterions $\left(\mathrm{Li}^{+}\right)$can migrate more [18]. This increases the polarisability of the particles and their interaction and hence increase the structure formation and leads to the growth of the ERE [8] [18].

\subsection{Fluctuations in Yield Stress at the Maximum}

Fluctuations in the yield stress at the maximum are not well understood. Silica particles are negatively charged. If a system with negatively charged disperse phase is held for a short period of time in a high-voltage field, a layer slightly 
enriched with the dispersion medium could be formed at the negatively charged electrode. Upon recharging of the electrode and starting the deformation, fluctuations of the yield stress may be observed. Recharging in the electric field can occur due to charging of metallic particles, recharging of hydrated particles as a result of electron chemical reactions of the decomposition of water on the electrodes (most likely mechanism in the systems studied), or recharging without contact of the particles with the electrode [4]. The alternative explanation of fluctuations could be associated with the radial movement of a layer of dispersion medium in the electric field due to deformation of the system and multiple transitions through the ultimate strength at zones inside the system [1].

\subsection{Drop in ERE at Higher Electric Fields}

Strong electric fields can cause alignment of the spherical particles. On applying the shear stress, the particles could easily "slip away" from each other because of the possible "uniform" alignment of the silica particles. The stronger the electric field, the more the particles are aligned and the easier it becomes for the structure breakdown (i.e. system yielding) [18]. The other possibility is that the systems studied contained water. Higher fields could cause the appearance of free water in the dispersion by some mechanism which is not understood. This could result in the sharp drop of the electrical resistance of the chains. The electrical conductance drops sharply because of the free water that causes water "gaskets" to form in the places of contact between the particles [1]. Charge exchange between particles lowers the polarisation and could lead to a decrease of the ERE. Finally, the drop could be due to the weakening of the particle interaction at higher electrical fields by the increased migration of particles.

\section{Conclusion}

The silica samples have shown that the water content increases the ERE. Both water content and small but finite conducting ions are important for the ERE to occur. Increasing the water content increases the ERE. There is a tendency to electric saturation or electric breakdown of the silica dispersions and a pronounced shift of the electric saturation to lower electric fields as the water content increases. As observed in this work, there is a possibility that high electric fields may lead to formation of shorter chains, which would lower the values of the shear stress.

\section{References}

[1] Sheng, P., Wen, W. and Huang, X. (2008) Electrorheological Fluids: Structures and Mechanisms. Soft Matter, 4, 200-210. https://doi.org/10.1039/B710948M

[2] Winslow, W.M. (1949) Induced Fibration of Suspensions. Journal of Applied Physics, 20, 1137-1140. https://doi.org/10.1063/1.1698285

[3] Inasaki, A.I. (1997) Application of Electrorheological Fluid Dampers to Machine Tool Elements. CIRP Annals-Manufacturing Technology, 46, 309-312.

[4] Gawade, S.S. and Jadhav, A.A. (2012) A Review on Electrorheological (ER) Fluids 
and Its Applications. International Journal of Engineering Research \& Technology, 1, 1-7.

[5] Wen, W., Huang, X., Yang, S., Lu, K. and Sheng, P. (2003) The Giant Electrorheological Effect in Suspensions of Nanoparticles. Nature Materials, 2, 727-730. https://doi.org/10.1038/nmat993

[6] Monkman, G.J. (1991) Addition of Solid Structures to Electrorheological Fluids. Journal of Rheology, 35, 1385-1387. https://doi.org/10.1122/1.550237

[7] Bergna, H.E., Ed. (1994) The Colloid Chemistry of Silica. In: Comstock, J., Ed., Advances in Chemistry Series, Vol. 234, American Chemical Society, Washington DC. https://doi.org/10.1021/ba-1994-0234

[8] Tam, W.Y., Yi, G.H., Wen, W., Loy, M.M.T. and Sheng, P. (1997) New Electrorheological Fluid: Theory and Experiment. Physical Review Letters, 78, 2987-2990. https://doi.org/10.1103/PhysRevLett.78.2987

[9] Unal, H.I., Sahan, B. and Erol, O. (2012) Investigation of Electrokinetic and Electrorheological Properties of Polyindole Prepared in the Presence of a Surfactant. Materials Chemistry and Physics, 134, 382-391.

[10] Deinega, Y.F. and Vindogradov, G.V. (1984) Electric Fields in the Rheology of Disperse Systems. Rheologica Acta, 23, 636-651. https://doi.org/10.1007/BF01438804

[11] Brunn, P.O. and Abu-Jdayi, B. (2004) A Phenomenological Model of Electrorheological Fluids. Rheologica Acta, 43, 62-67.

https://doi.org/10.1007/s00397-003-0320-0

[12] Cabuka, M., Yavuza, M. and Unalb, H.I. (2016) Electrokinetic, Electrorheological and Viscoelastic Properties of Polythiophene-Graft-Chitosan Copolymer Particles. Colloids and Surfaces A: Physicochemical and Engineering Aspects, 510, 231-238.

[13] Puvanatvattana, T., Chotpattananont, D., Hiamtup, P., Niamlang, S., Kunanuruksapong, R., Sirivat, A. and Jamieson, A.M. (2008) Electric Field Induced Stress Moduli of Polythiophene/Polyisoprene Suspensions: Effects of Particle Conductivity and Concentration. Materials Science and Engineering C, 28, 119-128.

[14] Krztoń-Maziopa, A., Ciszewska, M. and Płocharski, J. (2005) Electrorheological Fluids Based on Polymer Electrolytes. Electrochimica Acta, 50, 3838-3842.

[15] Gehin, C., Persello, J., Charraut, D. and Cabane, B. (2004) Electrorehological Properties and Microstructure of Silica Suspensions. Journal of Colloid and Interface Science, 273, 658-667.

[16] Belza, T., Pavlinek, V., Saha, P. and Quadrat, O. (2007) Electrorheological Properties of Suspensions of Silica Nanoparticles Modified by Urea and $N, N$-Dimethylformamide. Colloids and Surfaces A: Physicochemical and Engineering Aspects, 297, 142-146.

[17] Chelif, K., Moalla, S., Sassi, S. and Zarrouk, H. (2007) Electrorheological Response of Modified Silica Suspensions. Journal of the European Ceramic Society, 27, 11991202.

[18] Cho, M.S., Choi, H.J. and Jhon, M.S. (2005) Shear Stress Analysis of a Semiconducting Polymer Based Electrorheological Fluid System. Polymer, 46, 11484-11488.

[19] Hsu, J.P., Tai, Y.H. and Yeh, L.H. (2012) Importance of Temperature Effect on the Electrophoretic Behaviour of Charge-Regulated Particles. Langmuir, 28, 1013-1019. https://doi.org/10.1021/la203245n 
Submit or recommend next manuscript to SCIRP and we will provide best service for you:

Accepting pre-submission inquiries through Email, Facebook, LinkedIn, Twitter, etc. A wide selection of journals (inclusive of 9 subjects, more than 200 journals)

Providing 24-hour high-quality service

User-friendly online submission system

Fair and swift peer-review system

Efficient typesetting and proofreading procedure

Display of the result of downloads and visits, as well as the number of cited articles Maximum dissemination of your research work

Submit your manuscript at: http://papersubmission.scirp.org/

Or contact msa@scirp.org 